\title{
Intensified glucose lowering in type 2 diabetes. Reply to Czupryniak L, Szymańska-Garbacz E, Pawłowski M et al. [letter], Aizawa $\mathbf{T}$ and Funase $\mathrm{Y}$ [letter] and DeVries JH [letter]
}

\author{
J. S. Yudkin • B. Richter • E. A. M. Gale
}

Received: 3 November 2010 / Accepted: 9 November 2010 /Published online: 17 December 2010

(C) Springer-Verlag 2010

Keywords Cardiovascular disease - Glycaemic control . Guidelines · Type 2 diabetes · Risk factors · Risk reduction

\section{Abbreviations \\ ACCORD Action to control cardiovascular risk in diabetes \\ CONTROL Collaborators on trials of lowering glucose UKPDS UK Prospective diabetes study}

To the Editor: We are grateful to Czupryniak and colleagues [1] and to Aizawa and Funase [2] for questioning the applicability of our conclusions [3] to different population subgroups. We will focus our response on the issue of pharmacological interventions for prevention of macrovascular and microvascular complications, as we were not attempting to address matters such as lifestyle interventions, reversal of impaired glucose tolerance, or pancreatic beta cell mass [2].

\section{J. S. Yudkin}

Department of Medicine, University College London,

London, UK

J. S. Yudkin $(\square)$

28, Huddleston Road,

London N7 0AG, UK

e-mail: j.yudkin@ucl.ac.uk

B. Richter

Cochrane Metabolic and Endocrine Disorders Review Group,

Heinrich-Heine University,

Düsseldorf, Germany

\section{E. A. M. Gale}

Diabetes and Metabolism, Learning and Research, University of Bristol,

Bristol, UK
Both respondents suggest that there may be benefit to be gained from intensified glucose control in subgroups of patients with type 2 diabetes - younger patients, those with a shorter disease duration, and those without evidence of complications. The conclusions of a meta-analysis can be considered applicable only to individuals with similar characteristics - in this instance, a population with type 2 diabetes of mean age 62 years, mean duration of diabetes 7.3 years, mean BMI $29.7 \mathrm{~kg} / \mathrm{m}^{2}$ and mean $\mathrm{HbA}_{1 \mathrm{c}}$ level $7.9 \%$. Czupryniak et al. [1] quote the Collaborators on Trials of Lowering Glucose (CONTROL) Group metaanalysis [4] as showing benefits 'in those whose $\mathrm{HbA}_{1 \mathrm{c}}$ was lower when entering the intensive treatment arm, those who were diagnosed with type 2 diabetes less than 5 years before and those who were free from micro- and macrovascular complications', a possibility which we discussed in our paper [3]. The issue of post hoc subgroup analysis of clinical trials or meta-analyses needs considerable care. The CONTROL Group did indeed analyse the impact of intensified glycaemic control on major cardiovascular events in six pre-specified subgroups, and only that for a history of macrovascular disease achieved a level of significance, set by the authors at the $5 \%$ level $(p=0.04)$. We have previously highlighted the caution needed in interpreting such analyses [5], quoting the Second International Study of Infarct Survival (ISIS-2) Collaborative Group [6], who noted that aspirin was without statistical benefit on 35 day mortality after myocardial infarction either in patients with diabetes or in those born under the star signs Gemini or Libra. Subgroup analyses, unless adequately powered, should be considered as hypothesis generating rather than as defining populations that will benefit, particularly if they were not defined a priori and if no formal interaction test was performed. Moreover, when such subgroup analysis is performed according to responses 
to treatment, like that for the Action to Control Cardiovascular Risk in Diabetes (ACCORD) study [7], as the authors themselves note, 'These observations must be interpreted cautiously, because the analyses were not defined before treatment was started, the period of follow-up was shorter than planned, and the results could have been influenced by many post-randomisation factors.'

DeVries [8] correctly notes that the (clinical) heterogeneity of included trials makes meta-analysis difficult. It is indeed the case that in the ACCORD study a greater proportion of patients were taking rosiglitazone in the intensive therapy group $(91.2 \%)$ than in the standard therapy group $(57.5 \%)$ [9]. The same was true in the Action in Diabetes and Vascular Disease: Preterax and Diamicron Modified Release Controlled Evaluation (ADVANCE) study (16.8\% vs $10.9 \%$ on thiazolidinediones) [10], though this was not the case in the Veterans Affairs Diabetes Trial (VADT) $(100 \%$ in each) [11]. We have explored the contention that there were differences in effects between the UK Prospective Diabetes Study (UKPDS) and the later studies, and found no statistically significant heterogeneity in overall pooled effect estimates for cardiovascular events [12]. The follow-up investigation of the UKPDS should be regarded as observational in design. Because only $36.2 \%$ of the original randomised cohort provided data for complete post-trial monitoring, confirmatory evidence cannot be inferred from the findings of this study, and numbers needed to treat from observational studies have to be interpreted with extreme caution. We suggest that when young people are facing decades of treatment with interventions for which the benefits and hazards are unknown, it is essential that these people should not be exposed to the inconvenience and potential risks of intensified therapy as a consequence of extrapolations from observational studies.

All responses to our Editorial attempt to explore whether intensified glycaemic control might result in a larger reduction in relative risk in certain types of patients. Yet one of the main points of our analysis was to suggest the need for a greater emphasis on absolute, rather than relative, risk reduction. While younger patients, those with a shorter disease duration, and those without comorbidities may yet definitively be shown to benefit more, in relative terms, from intensified glucose lowering, such patients are at lower absolute risk, thus affecting the absolute benefit they may reap. In the UKPDS [13], 10 years of intensified glycaemic control in newly diagnosed patients of mean age 53 years produced reductions of $2.1 \%$ in cardiovascular events, $0.6 \%$ in blindness and $0.2 \%$ in renal failure, which implies that 34 out of 35 people initiated on such a regimen for 10 years will not be prevented from sustaining such an outcome during this time. We suggest that informed consent from patients requires presentation of the possible benefits, and risks, of treatment in similar format.

Duality of interest The authors declare that there is no duality of interest associate with this manuscript.

\section{References}

1. Czupryniak L, Szymańska-Garbacz E, Pawłowski M, SaryuszWolska M, Loba J (2011) Intensified glucose lowering in type 2 diabetes: time for a bolder reappraisal. Diabetologia. doi:10.1007/ s00125-010-2011-6

2. Aizawa T, Funase Y (2011) Intervention at the very early stage of type 2 diabetes. Diabetologia. doi:10.1007/s00125-010-1997-0

3. Yudkin JS, Richter B, Gale EAM (2010) Intensified glucose lowering in type 2 diabetes: time for a reappraisal. Diabetologia 53:2079-2085

4. Turnbull FM, Abraira C, Anderson RJ et al (2009) Intensive glucose control and macrovascular outcomes in type 2 diabetes. Diabetologia 52:2288-2298, Erratum 52:2470

5. Yudkin JS (1996) Assessing the evidence on aspirin in diabetes mellitus. Gemini or Libra; lumping or splitting; surrogate or hard; low or high; interventionist or nihilist. Diabetologia 39:1407-1408

6. ISIS-2 (Second International Study of Infarct Survival) Collaborative Group (1988) Randomised trial of intravenous streptokinase, oral aspirin, both, or neither among 17,187 cases of suspected myocardial infarction: ISIS-2. Lancet 332:349-360

7. Riddle MC, Ambrosius WT, Brillon DJ, Buse JB, Byington RP, Cohen RM, Goff DC Jr, Malozowski S, Margolis KL, Probstfield JL, Schnall A, Seaquist ER, Action to Control Cardiovascular Risk in Diabetes Investigators (2010) Epidemiologic relationships between $\mathrm{A} 1 \mathrm{C}$ and all-cause mortality during a median 3.4-year follow-up of glycemic treatment in the ACCORD Trial. Diab Care 33:983-990

8. DeVries JH (2011) Intensified glucose lowering in type 2 diabetes: don't throw the baby out with the bathwater. Diabetologia. doi:10.1007/s00125-010-1991-6

9. Action to Control Cardiovascular Risk in Diabetes (ACCORD) Study Group (2008) Effects of intensive glucose lowering in type 2 diabetes. N Engl J Med 358:2545-2559

10. The ADVANCE Collaborative Group (2008) Intensive blood glucose control and vascular outcomes in patients with type 2 diabetes. N Engl J Med 358:2560-2572

11. Duckworth W, Abraira C, Moritz T, VADT Investigators et al (2009) Glucose control and vascular complications in veterans with type 2 diabetes. N Engl J Med 360:129-139

12. Yudkin JS, Richter B (2010) Concerns about meta-analysis of glucose control and cardiovascular disease in type 2 diabetes. Ann Intern Med 152:63

13. UK Prospective Diabetes Study (UKPDS) Group (1998) Intensive blood-glucose control with sulphonylureas or insulin compared with conventional treatment and risk of complications in patients with type 2 diabetes (UKPDS 33). Lancet 352:837-853 